\title{
The shape of $\eta$ Carinae and LBV nebulae
}

\author{
A. Maeder and V. Desjacques
}

Geneva Observatory, 1290 Sauverny, Switzerland

e-mail: Andre.Maeder@obs.unige.ch; Vincent.Desjacques@obs.unige.ch

Received 19 December 2000 / Accepted 11 April 2001

\begin{abstract}
Stellar winds emitted by rotating massive stars may show two main components: firstly bipolar lobes with low density and fast wind, produced by the higher $T_{\text {eff }}$ and gravity at the poles (" $g_{\text {eff }}-$ effect"); secondly, an equatorial disc with a slow dense wind, produced by the stronger opacities at the equator (" $\kappa$-effect"). To see the possible role of this anisotropic wind on the shape of LBV nebulae, we calculate the distribution of the ejected matter in 2 simplified cases: 1) A brief shell ejection. We find that prolate and peanut-shaped hollow nebulae naturally form due to the $g_{\text {eff }}$-effect in rotating stars. 2) A constant wind for a long time. This produces prolate filled nebulae, with a possible strong disc when a bi-stability limit is crossed in the equatorial region. Thus, many features of the $\eta$ Carinae and LBV nebulae are accounted for by the anisotropic ejection from rotating stars.
\end{abstract}

Key words. $\eta$ Carinae - massive stars - LBV stars - mass loss

\section{Introduction}

The HST picture of the $\eta$ Carinae nebula (J. Morse and K. Davidson, STScI PRC96-23a) shows two big symmetrical lobes and a disk-shaped skirt around the star (Davidson \& Humphreys 1997; Davidson et al. 1997). These authors find that the lobes were created during the "Great Eruption" of 1843 , during which $\sim 1$ to $3 M_{\odot}$ were ejected forming the Homunculus Nebula, with a total energy of $\sim 10^{49.5}$ ergs (Humphreys 1999). The kinematics confirms that it is a bipolar outflow (Nota 1999), and the study of their limb darkening shows that the lobes are hollow (Davidson et al. 1997). The mass in the skirt amounts to 0.1 to $0.2 M_{\odot}$, it is still unclear whether it is resulting from the 1843 or later events. A massive cold torus of about $15 M_{\odot}$ has been found out in the equatorial plane (Morris et al. 1999). Peanut-shaped nebulae and lobes are present around several other LBV stars (Nota 1999).

Spectral variations with a period of $5.5 \mathrm{yr}$ have led to the suggestion that $\eta$ Carinae is a binary system (Damineli 1996; Lamers et al. 1998; Damineli et al. 2000). However, the periodicity is not as strict as claimed, with shifts of 3 monthes between two successive cycles, which casts some doubt on the binary hypothesis (Smith et al. 2000)

Numerous models for the $\eta$ Carinae nebula have been proposed (e.g. see Schulte-Ladbeck 1997; Hillier 1997). Several ones invoke collisions of winds emitted at various

Send offprint requests to: A. Maeder, e-mail: Andre.Maeder@obs.unige.ch evolutionary stages, such as the interaction of an isotropic fast wind with a previous slow equatorially enhanced wind (Kwok et al. 1978; Frank et al. 1995). The opposite scenario of an aspherical fast wind, expanding into a previously deposited slow spherical wind has been studied by Frank et al. (1998). This model recovers several features, but not the equatorial skirt. Langer (1998), Langer \& Heger (1998) consider that the LBV outbursts occur when the outwards centrifugal and radiation forces cancel gravity at the equator. Langer et al. (1999) develop a model where a slow, dense, equatorial wind is followed by a fast and almost spherical wind and this leads to very representative simulations of the Homunculus Nebula. The models are using the wind compressed disc model of Bjorkman \& Cassinelli (1993).

Owocki et al. (1993) have emphasized that the account of the von Zeipel theorem (1924) drastically modifies the wind distribution around a rotating star. The properties of stellar winds ejected by rotating stars have been studied (Maeder 1999; Maeder \& Meynet 2000), in particular the changes of the mass loss rates by rotation and the latitudinal distribution of the mass loss. Here, we examine the distribution of the anisotropic wind around rotating star models corresponding to $\eta$ Carinae. The noticeable result is that there are bi-polar components and a disc naturally arising in the wind. Interactions of the lobes and discs emitted at various phases during the back and forth motions of an LBV in the HR diagram may further shape the nebulae (cf. Langer et al. 1999). 


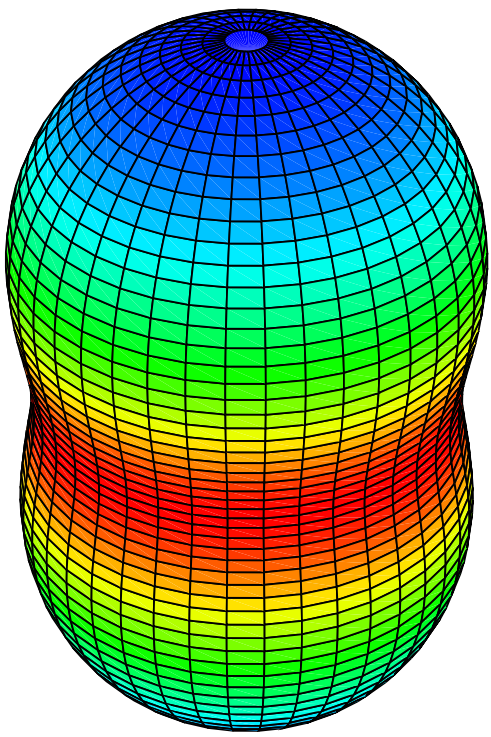

Fig. 1. The mass fluxes around a rotating star like $\eta$ Carinae for a ratio of the angular velocity to the break-up angular velocity $\omega=0.80$, assuming a polar $T_{\text {eff }}=30000 \mathrm{~K}$.

\section{The anisotropic mass loss}

In rotating stars with high radiation pressure, the surface shape is also described by the Roche model. The total gravity is the sum of the gravitational, centrifugal and radiative accelerations: $\boldsymbol{g}_{\text {tot }}=\boldsymbol{g}_{\text {eff }}+\boldsymbol{g}_{\text {rad }}=\boldsymbol{g}_{\text {grav }}+\boldsymbol{g}_{\text {rot }}+$ $\boldsymbol{g}_{\text {rad }}$. The local flux at colatitude $\vartheta$ is proportional to the effective gravity $\boldsymbol{g}_{\text {eff }}$ according to the von Zeipel theorem

$\boldsymbol{F}(\vartheta)=-\frac{L}{4 \pi G M\left(1-\frac{\Omega^{2}}{2 \pi G \rho_{\mathrm{m}}}\right)} \boldsymbol{g}_{\mathrm{eff}}(\vartheta)$

where we ignore a small term $(\sim 1 \%)$ due to differential rotation. The correct Eddington factor $\Gamma_{\Omega}(\vartheta)$ in a rotating star depends on rotation (Maeder \& Meynet 2000).

$\Gamma_{\Omega}(\vartheta)=\frac{\kappa(\vartheta) L}{4 \pi c G M\left(1-\frac{\Omega^{2}}{2 \pi G \rho_{\mathrm{m}}}\right)}$,

where $\kappa(\vartheta)$ is the local opacity. One has

$\boldsymbol{g}_{\mathrm{tot}}=\boldsymbol{g}_{\mathrm{eff}}\left[1-\Gamma_{\Omega}(\vartheta)\right]$.

The relation $\boldsymbol{g}_{\text {tot }}=0$ gives the critical velocity. The expression $v_{\text {crit }}^{2}=\frac{G M}{R}(1-\Gamma)$ often used for massive stars only applies if the star is uniformly bright. If not, Eq. (3) has two roots which need to be discussed (Maeder \& Meynet 2000). The result is that, due to the von Zeipel theorem, $v_{\text {crit }}$ only slowly tends towards zero for high Eddington factor.

The distribution of the mass loss by unit surface at colatitude $\vartheta$ over the surface of a rotating star is

$\frac{\Delta \dot{M}(\vartheta)}{\Delta \sigma} \simeq A\left[\frac{L(P)}{4 \pi G M\left(1-\frac{\Omega^{2}}{2 \pi G \rho_{\mathrm{m}}}\right)}\right]^{\frac{1}{\alpha}} \frac{g_{\mathrm{eff}}}{\left[1-\Gamma_{\Omega}\right]^{\frac{1}{\alpha}-1}}$ where $\Gamma_{\Omega}$ is given by Eq. (2) for electron scattering opacity. We have $A=(k \alpha)^{\frac{1}{\alpha}}\left(\frac{1-\alpha}{\alpha}\right)^{\frac{1-\alpha}{\alpha}}$. The parameter $\alpha$ is a so-called force multiplier, which characterizes the intensities and distribution of the strengths of the spectral lines. At some $T_{\text {eff }}$, the ionisation equilibrium of the stellar wind is changing rather abruptly and so does the opacity of the plasma. Consequently, the values of the force multipliers undergo rapid transitions, e.g. $\alpha=0.54$ for $\log T_{\text {eff }}$ $\geq 4.342, \alpha=0.30$ for $\log T_{\text {eff }}$ between 4.342 and 4.00 and $\alpha=0.20$ for $\log T_{\text {eff }} \leq 4.00$ (Lamers et al. 1995; Lamers 1997; see also Kudritzki \& Puls 2000). Such transitions of the wind properties, which still have some uncertainties, are called bi-stability limits. Since $T_{\text {eff }}$ is decreasing from pole to equator, one has the corresponding variations of the force multipliers and thus of the mass loss fluxes from pole to equator.

Equation (4) shows that two main effects may enhance the mass ejection. The first one is the higher gravity at the pole which makes it hotter, this is the " $g_{\text {eff-effect". The }}$ second is the " $\kappa$-effect", it is due to the rapid growth of the opacity below a bi-stability limit. The result is a lower value of $\alpha$ in the equatorial regions, if they become cool enough; this favours strong equatorial ejections.

We consider the case of $\eta$ Carinae, with a mass of $\sim 100 M_{\odot}$, a luminosity of $\sim 10^{6.5} L_{\odot}$, an Eddington factor $\Gamma=0.823$ for electron scattering opacity, and a $T_{\text {eff }} \simeq 30000 \mathrm{~K}$ or a few thousands degrees less at minimum (Humphreys 1988; Davidson et al. 1997). Rotation is defined by $\omega$, the fraction of the angular velocity at break-up, i.e. $\omega^{2}=\frac{\Omega^{2} R_{\mathrm{eb}}^{3}}{G M}$. Figure 1 shows the distribution of the mass fluxes for $\omega=0.80$ and a reference polar $T_{\text {eff }}=30000 \mathrm{~K}$. We notice the prolate distribution with a peanut-shape, which results from the $g_{\text {eff- }}$-effect. For higher values of $\omega$, the peanut-shape is more and more pronounced, while for lower $\omega$ we have a prolate spheroid.

When a bi-stability limit is crossed at some latitude on the rotating star, the $\dot{M}$-rates are strongly enhanced at lower latitudes by the $\kappa$-effect. A crossing of the bistability limit is favoured by fast rotation and a lower choice of the reference $T_{\text {eff }}$. Figure 2 shows, as an example, the mass flux for $\omega=0.8$ as in Fig. 1, but for $T_{\text {eff }}=$ $25000 \mathrm{~K}$. We notice the presence of a strong equatorial ejection. The wider the latitude range with $T_{\text {eff }}$ below the bistability limit, the thicker the disk.

\section{The shape of the nebulae}

The shape of the nebulae is given by the distribution of matter at some time around the ejecting star. For purpose of simplicity, we consider here two simplified cases: a) a short time ejection, b) a continuous ejection.

\subsection{Short time ejection}

We assume that the geometry $r(t, \vartheta)$ at time $t$ of the shell ejected at $t_{0}$ is determined by the coasting velocity, while Eq. (4) determines the amount of matter at a given 


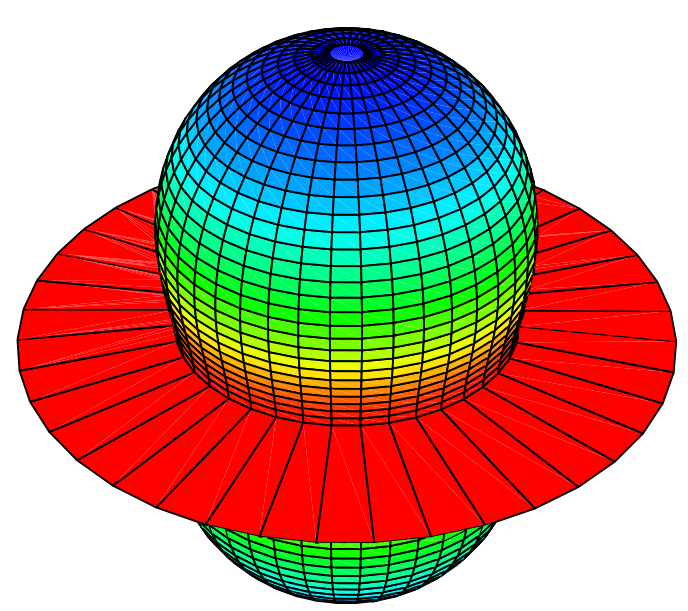

Fig. 2. The mass flux around a rotating star with the mass and luminosity of $\eta$ Carinae for $\omega=0.80$ and a polar $T_{\text {eff }}=$ $25000 \mathrm{~K}$.

position in the shell. Thus, one has (cf. Maeder 1999)

$$
\begin{aligned}
& r(t, \vartheta)=v_{\infty}(r, \vartheta)\left(t-t_{0}\right) \quad \text { with } \\
& v_{\infty}(r, \vartheta) \simeq \frac{\alpha}{1-\alpha} g_{\text {eff }}^{\frac{1}{2}} r(\vartheta)^{\frac{1}{2}}\left(1-\Gamma_{\Omega}\right)^{\frac{1}{2}}
\end{aligned}
$$

Figure 3 shows the distribution of matter for a short time ejection for a case with $\omega=0.90$. If there is no bi-stability limit crossed, this shape is the same for all kinds of stars with a given $\omega$ (cf. Eq. (6)). The nebula is hollow and shows a peanut shape with an equatorial pinch. For lower values of $\omega$, the shape is a prolate spheroid; for higher $\omega$, the equatorial pinch is even more marked. At a given $\omega$, the peanut shape is more pronounced for the $\dot{M}$ distribution than for the $v_{\infty}$ distribution, since the first one depends on $g_{\text {eff }}$, while the second one depends on $g_{\text {eff }}^{\frac{1}{2}}$. Such a shape well corresponds to that of many LBV nebulae (Nota 1999).

\subsection{Continuous ejection}

We may also assume the wind to be constant in time, and for a long time. On each radial line, the density decreases like $r^{-2}$. Thus, the lines $r(\vartheta)$ of constant densities are given by

$$
\begin{aligned}
& r(\vartheta)=\left[\frac{\rho\left(r_{0}, \vartheta\right)}{\rho(r, \vartheta)}\right]^{\frac{1}{2}} r_{0}(\vartheta) \text { with } \\
& \rho\left(r_{0}, \vartheta\right) \simeq(1-\alpha)^{\frac{1}{\alpha}} \frac{g_{\mathrm{eff}}^{\frac{1}{2}}}{r(\vartheta)^{\frac{1}{2}}\left[1-\Gamma_{\Omega}\right]^{\frac{1}{\alpha}-\frac{1}{2}}} .
\end{aligned}
$$

This model gives centrally filled nebulae. Figure 4 illustrates the iso-density lines in the case corresponding to Fig. 1. Since, the iso-density lines behave like $g_{\text {eff }}^{\frac{1}{4}}$, the variations of $g_{\text {eff }}$ have smaller effects and the peanut shape is absent. We notice a strong equatorial enhancement due to the lower $\alpha$ in these regions. In real cases, if a sudden ejection is superposed on a more continuous wind, we may have some compound of the hollow peanut shell as in

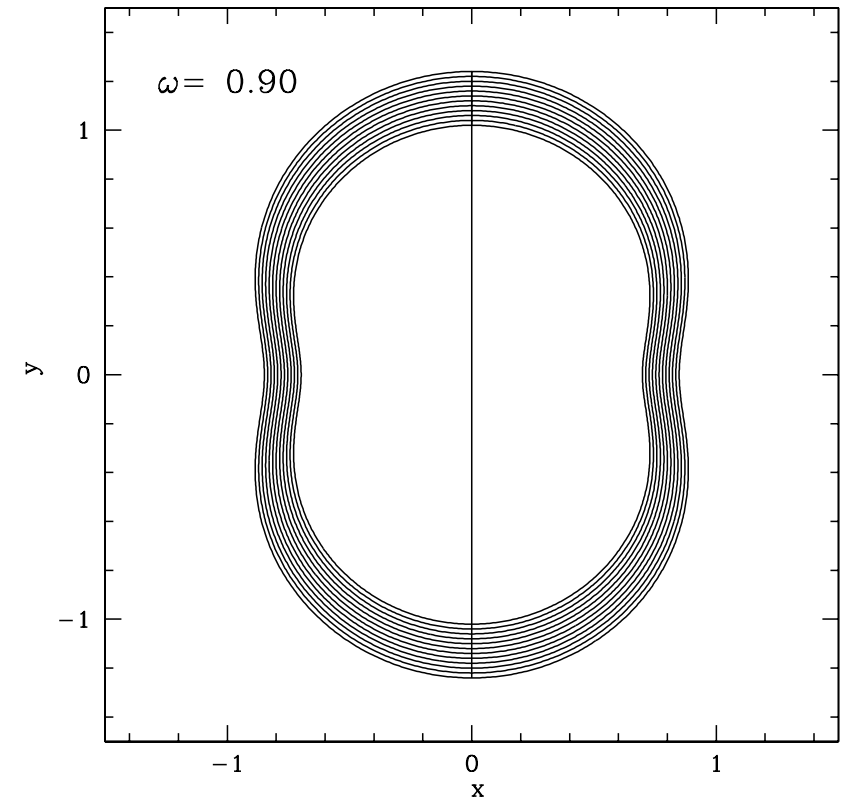

Fig. 3. Spatial distribution of the matter for a short ejection around a star with $\omega=0.90$. The vertical line shows the direction of the rotation axis.

Fig. 3 and of the filled nebula with a disc as in Fig. 4, well corresponding to the various possible shapes of the LBV nebulae and $\eta$ Carinae. Interestingly enough, the group of the B[e] stars (Zickgraf 1999) shows a two-component stellar wind with a hot, highly ionized, fast wind at the poles and a slow dense disk-like wind at the equator.

Figures 3 and 4 only give two schematic examples of nebulae. Certainly, a kind of catalogue is to be made of all possible sorts of nebulae arising from Eq. (4). This is a problem with many parameters:

- Stellar rotation given by $\omega$;

- Reference polar $T_{\text {eff }}$ of the star;

- Eddington factor $\Gamma$;

- Opacities, bi-stability limits, opacity peaks, etc.;

- Particular history of mass ejection $\dot{M}(t)$;

- Wind-wind interaction (cf. Langer et al. 1999).

Certainly many interesting situations may occur. For example, a B-star with a polar $T_{\text {eff }}$ not so much above the bi-stability limit could give rise to a substantial equatorial ejection and a disc formation, even if the star is not at break-up!

Finally, we may wonder whether LBV stars have fast rotation velocities. We see two possibilities. a) If due to a small magnetic field rotation keeps solid body (Langer 1998), for most non-zero initial velocities the star may reach the break-up velocity near the end of the MS. b) Models with differential rotation also show that very massive stars may reach the so-called $\Omega \Gamma$-limit, i.e the break-limit function of rotation and of the Eddington factor (Maeder \& Meynet 2000). In particular, after the end of the MS phase, the stars do a bluewards hook in the HR diagram; also after an outburst, they may reach fast 


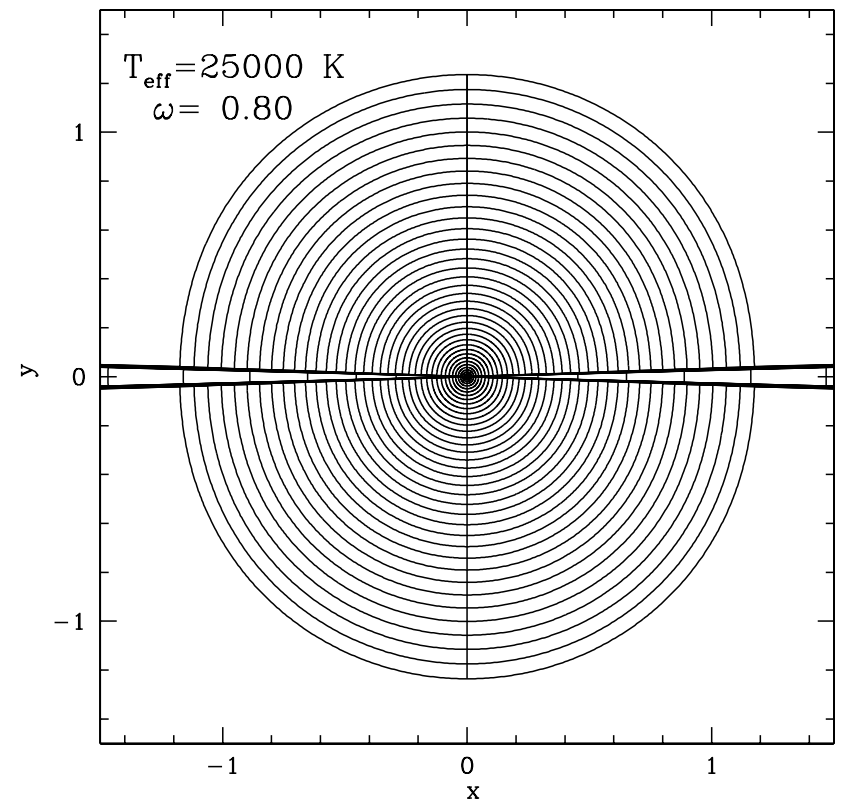

Fig. 4. Iso-density lines for a continuous ejection around a star with $\omega=0.80$. The density is strongly enhanced in the equatorial plane.

velocities as a result of the contraction of the outer layers.

\section{Conclusions}

Rotation produces strongly anisotropic mass loss in massive stars. Two schematic cases of winds have been considered. The occurence of a peanut-shaped nebula with bi-polar lobes and an equatorial disc may naturally result from the $g_{\text {eff }}$ and $\kappa$-effects. Without particular wind interactions and collisions, the wind distribution already possesses several important features of $\eta$ Carinae and of other LBV stars.

The critical question is whether the polar lobes and the skirt in $\eta$ Carinae result directly from the anisotropic mass ejection (cf. Figs. 3 and 4) or whether they result from the interaction of the successive anisotropic winds. Whatever the answer, the anisotropic ejection with polar lobes and an equatorial disc, as shown here, is an essential part of the game.

Acknowledgements. A. M. expresses his best thanks to Dr. Norbert Langer and to Dr. Georges Meynet for their very helpful and constructive comments, and to Raoul Behrend for his help in numerical modelling.

\section{References}

Bjorkman, J. E., \& Cassinelli, J. P. 1993, ApJ, 409, 429

Damineli, A. 1996, ApJ, 460, L49

Damineli, A., Kaufer, A., Wolf, B., et al. 2000, ApJ, 528, L101

Davidson, K., \& Humphreys, R. M. 1997, ARA\&A, 35, 1

Davidson, K., Ebbets, D., Johansson, S., Morse, J. A., \& Hamann, F. W. 1997, AJ, 113, 335

Davidson, K., Gull, T. R., Humphreys, R. M., et al. 1999, AJ, 118,1777

Frank, A., Balick, B., \& Davidson, K. 1995, ApJ, 441, L77

Frank, A., Ryu, D., \& Davidson, K. 1998, ApJ, 500, 291

Hillier, J. 1997, ASP Conf. Ser., 120, 287

Humphreys, R. M. 1992, in Physics of Luminous Blue Variables, IAU Coll. 113, ed. K. Davidson, et al. (Kluwer), 3

Humphreys, R. M. 1999, Lect. Notes Phys., 523, 243

Kudritzki, R. P., \& Puls, J. 2000, ARA\&A, 38, 613

Kwok, S., Purton, C. R., \& Fitzgerald, P. M. 1978, ApJ, 210, L125

Lamers, H. 1997, ASP Conf. Ser., 120, 83

Lamers, H., Livio, M., Panagia, N., \& Walborn, N. 1998, ApJ, 505, L131

Lamers, H., Snow, T. P., \& Lindholm, D. L. 1995, ApJ, 455, 269

Langer, N. 1998, A\&A, 329, 551

Langer, N., \& Heger, A. 1998, ASP Conf. Ser., 131, 76

Langer, N., Garcia-Segura, G., \& Mac Low, M. M. 1999, ApJ, 520, L49

Maeder, A. 1992, in Instabilities in evolved super- and hypergiants, ed. C. de Jager, \& H. Nieuwenhuijzen (North Holland), 138

Maeder, A. 1999, A\&A, 347, 185

Maeder, A., \& Meynet, G. 2000, A\&A, 361, 159

Meynet, G., \& Maeder, A. 2000, A\&A, 361, 101

Morris, P. W., Waters, L. B. F. M., Barlow, M. J., et al. 1999, Nature, 402, 502

Owocki, S. P., Gayley, K. G., \& Cranmer, S. R. 1998, ASP Conf. Ser., 131, 237

Nota, A., \& Clampin, M. 1999, ASP Conf. Ser., 120, 303

Nota, A. 1999, Lect. Notes Phys., 523, 62

Schulte-Ladbeck, R. E. 1997, ASP Conf. Ser., 120, 355

von Zeipel, H. 1924, MNRAS, 84, 665

Smith, N., Morse, J. A., Davidson, K., \& Humphreys, R. M. 2000, AJ, 120, 920

Zickgraf, F. J. 1999, Lect. Notes Phys., 523, 40 